\section{Hämoglobin, glykiertes}

\author{
K. J. Lackner ${ }^{1}$ und D. Peetz ${ }^{2}$ \\ ${ }^{1}$ Institut für Klinische Chemie und Laboratoriumsmedizin, \\ Universitätsmedizin Mainz, Mainz, Deutschland \\ ${ }^{2}$ Institut für Labormedizin, Helios Klinikum Berlin-Buch, \\ Berlin, Deutschland
}

Synonym(e) Glykohämoglobin

Englischer Begriff glycated hemoglobin

Definition Glykierungsprodukte von Hämoglobin mit unterschiedlichen Kohlenhydraten, vornehmlich Glukose.

Beschreibung Wie andere Proteine wird Hämoglobin nichtenzymatisch glykiert. Freie Aminogruppen für diese Reaktion sind an den N-terminalen Aminosäuren der Globinketten und an mehreren Lysinresten verfügbar. Da die Reaktion in erster Linie von der Konzentration der Kohlenhydrate abhängt, gibt die Konzentration von glykiertem Hämoglobin Aufschluss über den Glukosegehalt des Bluts während der Lebensdauer eines Erythrozyten. Zur Nomenklatur der glykierten Hämoglobine s. folgende Tabelle:

$\mathrm{Zu}$ beachten ist, dass $\mathrm{HbA}$ und $\mathrm{HbA}_{2}$ Hämoglobine unterschiedlicher Globinzusammensetzung bezeichnen, während $\mathrm{HbA}_{1}$ sich auf glykiertes $\mathrm{HbA}$ bezieht. Diese Inkonsequenz der Nomenklatur ist historisch entstanden. Die nicht mit Glukose glykierten Formen werden bei den selteneren Hämoglo-

\begin{tabular}{|c|c|c|c|}
\hline $\begin{array}{l}\text { Nichtglykiertes } \\
\mathrm{Hb}\end{array}$ & $\begin{array}{l}\text { Anteil } \\
(\%)\end{array}$ & $\begin{array}{l}\text { Glykierte } \\
\text { Formen }\end{array}$ & Kommentar \\
\hline \multirow[t]{5}{*}{$\mathrm{HbA}(\mathrm{a} 2, \mathrm{~b} 2)$} & \multirow[t]{5}{*}{ 95-97 } & $\mathrm{HbA}_{1}$ & $\begin{array}{l}\text { N-terminal an der } \beta \text {-Kette } \\
\text { glykiertes } \mathrm{HbA}\end{array}$ \\
\hline & & $\mathrm{HbA}_{1 \mathrm{a} 1}$ & $\begin{array}{l}\text { Mit Fruktose-1,6- } \\
\text { Bisphosphat }\end{array}$ \\
\hline & & $\mathrm{HbA}_{1 \mathrm{a} 2}$ & Mit Glukose-6-Phosphat \\
\hline & & $\mathrm{HbA}_{1 \mathrm{~b}}$ & Mit unbekanntem Partner \\
\hline & & $\mathrm{HbA}_{1 \mathrm{c}}$ & $\begin{array}{l}\text { Mit D-Glukose } \\
\text { (ca. } 75-80 \% \text { des } \mathrm{HbA}_{1} \text { ) }\end{array}$ \\
\hline $\mathrm{HbA}_{2}(\mathrm{a} 2, \mathrm{~d} 2)$ & $<3$ & $\mathrm{HbA}_{2 \mathrm{c}}$ & $\begin{array}{l}\text { N-terminal an der } \beta \text {-Kette } \\
\text { mit D-Glukose glykiertes } \\
\mathrm{HbA}_{2}\end{array}$ \\
\hline $\mathrm{HbF}(\mathrm{a} 2, \mathrm{~g} 2)$ & $<1$ & $\mathrm{HbF}$ & $\begin{array}{l}\text { N-terminal an der } \beta \text {-Kette } \\
\text { mit D-Glukose glykiertes } \\
\mathrm{HbF}\end{array}$ \\
\hline
\end{tabular}

binen $\mathrm{A}_{2}$ und $\mathrm{F}$ nicht berücksichtigt, weil sie quantitativ zum Gesamthämoglobin vernachlässigbar sind. Wenn neben dem N-terminal glykierten Hämoglobin auch an Aminogruppen von Lysinen glykierte Varianten detektiert werden, wird vom Gesamtglykohämoglobin gesprochen. Die Analytik glykierter Hämoglobine erfolgt meist mittels \ Ionenaustauschchromatographie, elektrophoretisch oder immunologisch und erfasst in der Regel nur die N-terminal glykierten Formen. Die verschiedenen Methoden machen sich Unterschiede in Ladung, Struktur oder Chemie zunutze. In den letzten Jahren hat sich die Bestimmung von $\mathrm{HbA}_{1 \mathrm{c}}(\triangleright$ Hämoglobin $\mathrm{A} 1 \mathrm{c})$ in der klinischen Routine gegenüber den anderen Methoden durchgesetzt (s. Tabelle). 